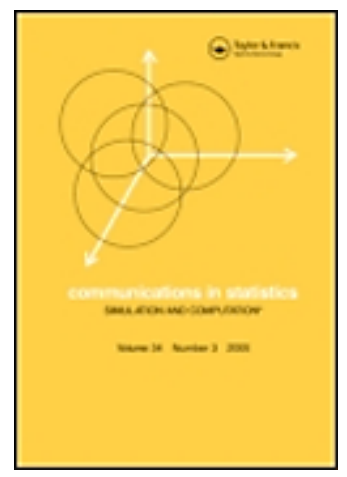

\title{
An empirical strategy to detect spurious effects in long memory and occasional-break processes
}

\begin{tabular}{|c|c|}
\hline Journal: & Communications in Statistics - Simulation and Computation \\
\hline Manuscript ID: & LSSP-2007-0059.R2 \\
\hline Manuscript Type: & Original Paper \\
\hline $\begin{array}{r}\text { Date Submitted by the } \\
\text { Author: }\end{array}$ & 27-Aug-2008 \\
\hline Complete List of Authors: & $\begin{array}{l}\text { Bisaglia, Luisa; Padova University } \\
\text { Gerolimetto, Margherita; Padova University }\end{array}$ \\
\hline Keywords: & Long memory, Occasional structural breaks, Break-free series \\
\hline Abstract: & $\begin{array}{l}\text { Long range dependence and structural changes in level } \\
\text { are intimely related phenomena and it is very difficult to separate } \\
\text { the two effects. } \\
\text { In this paper we present an empirical procedure to distinguish } \\
\text { between long memory and occasional-break processes. } \\
\text { An extensive Monte Carlo experiment illustrates the performance of } \\
\text { the procedure and an application } \\
\text { to real data is also included. }\end{array}$ \\
\hline \multicolumn{2}{|c|}{$\begin{array}{l}\text { Note: The following files were submitted by the author for peer review, but cannot be converted } \\
\text { to PDF. You must view these files (e.g. movies) online. }\end{array}$} \\
\hline bisaglia_gerolimetto_revised & 2.tex \\
\hline
\end{tabular}

\section{S ScholarONE" \\ Manuscript Central}




\title{
An empirical strategy to detect spurious effects in long memory and occasional-break processes
}

\author{
Luisa Bisaglia, Margherita Gerolimetto *
}

August 27, 2008

\begin{abstract}
Long range dependence and structural changes in level are intimely related phenomena and it is very difficult to separate the two effects. In this paper we present an empirical procedure to distinguish between long memory and occasional-break processes. An extensive Monte Carlo experiment illustrates the performance of the procedure and an application to real data is also included.
\end{abstract}

key words: Long memory, Occasional structural breaks, Break-free series

\section{Introduction}

Since the seminal papers of Granger and Joyeux (1980) and Hosking (1981) there has been a considerable interest in modelling strong persistence in time series. This interest is motivated by the analysis of many empirical time series whose autocorrelation function decreases to zero like a power function rather than exponentially and the spectral density diverges as the frequencies tend to zero.

Many authors have studied the problem of estimating the long memory parameter with both parametric and non/semi-parametric methods (see Beran, 1994, Percival and Walden, 2000 and Palma, 2007 for good reviews on this argument). However, recently (see, for example, Giraitis et al., 2001, Berkes et al., 2006 and the refences therein), it has been shown that inference on the long memory parameter and persistence tests are severely compromised in series which display occasional structural breaks, since these processes give the impression of persistence. In

*Department of Statistics, University of Padova, via C. Battisti 241, 35121 Padova, Italy, bisaglia@stat.unipd.it, gerolime@stat.unipd.it 
other words, neglecting structural changes causes a spurious over estimation of the long memory parameter, leading the reseacher to believe erroneously in a long memory data generating process (among the others, Granger and Hyung, 2004, Diebold and Inoue, 2001).

On the other hand, if an observed time series follows a fractionally integrated model, then change point tests may indicate the presence of change points, when, in fact, there are no change. Indeed, when the Data Generated Process (hereafter, DGP) is fractionally integrated of order $d, I(d)$, with $0<d<0.5$ and no change, many well-known structural change tests are likely to suggest that changes have spuriously take place (Kuan and Hsu, 1998).

From an empirical point of view, Morana and Beltratti (2004) analyse the realized variance process for the DM/US\$ and Yen/US\$ exchange rates. They find that while long memory is evident in the actual process, a structural break analysis reveals that this feature is partially explained by unaccounted changes in regime. Hsu (2005) analyses monthly G7 inflation rates and suggests that for Germany and Japan the long memory phenomenon may just be a consequence of structural change. The same findings apply to other countries, whose inflation rates may exhibit both long memory and structural change. Gil-Alana (2004) analyses annual data on German real GDP and shows that the series may be well described in terms of fractional models with a structural slope break due to World War II.

Moreover, the importance of distinguishing between long memory and occasional-break models is justified also in the perspective of forecasting (Diebold and Inoue, 2001, Gabriel and Martins, 2004).

With this paper we contribute in two directions. Firstly, we conduct an extensive Monte Carlo experiment to evaluate and compare the performance of various estimators of the long memory parameter under some occasional-break DGPs.

Secondly, we present an empirical strategy to detect the spurious effects caused by a misspecification of the model when the data show persistence. The procedure is based on the different behaviour of the long memory parameter estimators when applied to the original series and its break-free version. A further Monte Carlo experiment illustrates the performance of the strategy. We also apply the suggested strategy to the daily series of absolute S\&P500 stock returns to show with an empirical example how to implement the procedure.

In literature, to our knowledge, there are only a few proposals to separate the long memory from the occasional breaks effects (Ohanissian et al., 2004, Dolado et al., 2004, Teyssiere and 
Abry, 2006). However, they do not refer to the cases we consider.

The plan of the paper is as follows. In Section 2, we briefly introduce $I(d)$ processes. In Section 3 some models for occasional structural breaks are discussed. Section 4 describes a simple empirical strategy to deal with spurious effects. Section 5 describes our Monte Carlo experiments. In Section 6 we apply the proposed methodology to the S\&P 500 absolute stock returns series and Section 7 concludes.

\section{Structural breaks}

Following Granger and Hyung (2004) we describe three models with occasional breaks in mean, hence the number of breaks that can occur in a specific period of time is somehow bounded.

More formally, we assume, as in Granger and Hyung (2004), that the probability of breaks, $p$, converges to zero slowly as the sample size increases, i.e. $p \rightarrow 0$ as $T \rightarrow \infty$, yet $\lim _{T \rightarrow \infty} T p$ is a non-zero finite constant.

This implies that letting $p$ decrease with the sample size, realization tends to have just a finite number of breaks (see also Diebold and Inoue, 2001).

The three model we consider are the following:

1. Mean plus noise model (Chen and Tiao, 1990, Engle and Smith, 1999)

$$
\begin{aligned}
& y_{t}=m_{t}+\epsilon_{t}, \quad t=1, \ldots, T \\
& m_{t}=m_{t-1}+q_{t} \eta_{t}
\end{aligned}
$$

where $\epsilon_{t}$ is a noise variable ${ }^{1}$ and occasional level shifts, $m_{t}$, are controlled by two variables $q_{t}$ (date of breaks) and $\eta_{t}$ (size of jump). $\eta_{t}$ is an i.i.d. $N\left(0, \sigma_{\eta}^{2}\right)$ although the normality assumption can be dropped and $q_{t}$ is assumed to be an i.i.d. sequence of Bernoulli random variables such that $\operatorname{Pr}\left(q_{t}=1\right)=p$.

2. Markov switching model (Hamilton, 1989) The binomial model is characterized by sudden changes only, but the structural changes might also occur gradually. In this case a Markov switching model is more appropriate. Suppose $s_{t}$ is a latent random variable that can assume only values 0 or $1 . s_{t}$ is assumed to be a Markov chain, with transition probability $p_{i j}=\operatorname{Pr}\left(s_{t}=j / s_{t-1}=i\right)$. Then, it is possible to use a switching model for $q_{t}$ such that $q_{t}=0$ when $s_{t}=0$ and $q_{t}=1$ when $s_{t}=1$. In this specification a regime with $s_{t}=1$ represents a period of structural change, regardless of the value of $s_{t-1}$.

\footnotetext{
${ }^{1}$ Even if $\epsilon_{t}$ can be any short memory process, in this work we consider $\epsilon_{t} \sim$ i.i.d.N $\left(0, \sigma_{\epsilon}^{2}\right)$
} 
3. Stocastic Permanent Break (STOPBREAK) model (Engle and Smith, 1999)

This model bridges the gap between transience and permanence of the shocks. In the STOPBREAK process the long run impact of each obervation is time varying and stochastic. The formulation is as follows:

$$
\begin{aligned}
& y_{t}=m_{t}+\epsilon_{t} \\
& m_{t}=m_{t-1}+q_{t-1} \epsilon_{t-1}
\end{aligned}
$$

where $q_{t}=q\left(\left|\epsilon_{t}\right|\right)$ is non decreasing in $\left|\epsilon_{t}\right|$ and bounded by zero and one, so that bigger innovations have more permanent effects, and $\epsilon_{t}$ are i.i.d $N\left(0, \sigma_{\epsilon}^{2}\right)$, moreover $q_{t}=\frac{\epsilon_{t}^{2}}{\left(\gamma+\epsilon_{t}^{2}\right)}$ for $\gamma>0$. Therefore in the STOPBREAK process permanent shocks can be identified by their larger magnitude. With this approach the effects of shocks can fluctuate between transient and permanent. Typically, realizations of this process show periods of apparent stationarity punctuated by occasional mean shifts.

To estimate and test for multiple breaks at unknown dates one of the most used methods is the Bai-Perron (hereafter BP) procedure (Bai and Perron, 1998, 2003). It is based on a type test for the null hypothesis of no change versus a prespecified number of changes and also an alternative of an arbitrary number of changes (up to some maximum) as well as a procedure that allows one to test for the null hypothesis of, say, $l$ changes against the alternative hypothesis of $l+1$ changes. The latter is particularly useful in that it allows a specific to general modeling strategy to determine consistently the appropriate number of changes in the data. The tests can be constructed allowing different serial correlation in the errors, different distributions for the data and the errors across segments or imposing a common structure.

\section{$3 \quad$ Fractionally integrated processes}

There are various definitions of long memory processes. In particular, long memory can be expressed either in the time domain or in the frequency domain. In the time domain, a stationary discrete time series is said to be long memory if its autocorrelation function decays to zero like a power function. This definition implies that the dependence between successive observations decays slowly as the number of lags tends to infinity. On the other hand, in the frequency domain, a stationary discrete time series is said to be long memory if its spectral density is unbounded at low frequencies. 
In this paper we consider the fractionally integrated process, $I(d)$, independently introduced by Granger and Joyeux (1980) and Hosking (1981). Let $\epsilon_{t}$ be a white noise process such that $E\left[\epsilon_{t}^{2}\right]=\sigma^{2}$. The process $\left\{X_{t}, t \in \mathbf{Z}\right\}$ is said to be an $I(d)$ process with $d \in(-1 / 2,1 / 2)$, if it is stationary and satisfies the difference equation

$$
\Delta(B)\left(X_{t}-\mu\right)=\epsilon_{t}
$$

where $\Delta(B)=(1-B)^{d}=\sum_{j=0}^{\infty} \pi_{j} B^{j}$ with $\pi_{j}=\Gamma(j-d) /[\Gamma(j+1) \Gamma(-d)], \Gamma(\cdot)$ is the gamma function and $\mu$ is the mean of the process.

In the following we will concentrate on $I(d)$ processes with $d \in(0,1 / 2)$ : for this range of values the process is stationary, invertible and possesses long-range dependence. Moreover, we will assume for convenience and without loss of generality that $\sigma^{2}=1$ and $\mu=0$.

In literature several methods to estimate the long memory parameter $d$ have been proposed, see Palma (2007) for a comprehensive review.

To our knowledge, in the literature regarding the problem of discriminating between long memory and occasional-break processes only Gaussian semiparametric estimators like the GewekePorter Hudak (1983) method (hereafter GPH), the local Whittle method (Kunsch, 1987, Robinson, 1995b) or Lobato and Robinson's LM test (1998) are used. ${ }^{2}$ There are no results on the performance of other estimation methods, for this reason in the Monte Carlo study in Section 5 of this paper we will consider:

1. The R/S or rescaled adjusted range method (Hurst, 1951, Mandelbrot 1972, 1975, Mandelbrot and Taqqu, 1979);

2. The aggregate variance method (Taqqu et al., 1995);

3. Higuchi method (Higuchi, 1988);

4. Whittle method (Fox and Taqqu, 1986)

5. Robinson's modified version of the periodogram method (Robinson, 1995a).

The GPH method is also included in the Monte Carlo experiment with the role of benchmark. Indeed, Granger and Hyung (2004), Perron and Qu (2006) and Smith (2005) show analitically

\footnotetext{
${ }^{2}$ We thank a referee for reminding us of the existence of a strain of literature that treats the estimation of $d$ with wavelets (e.g. Jensen, 1999, Wang, 1999, Craigmile et al., 2005). In this framework Teyssiere and Abry (2006) consider also the issue of distinguishing between long memory and structural breaks but the models they study are different from the models analyzed in this paper.
} 
that, in hypothesis of occasional-break process, the GPH estimator is biased. Smith (2005) derives an approximation to this bias and proposes a modified version of the GPH estimator that takes into account this result. Bearing in mind the bias of the GPH estimator, we included also Robinson's version of the GPH (R-GPH, herafter) since it triggers out the first frequencies of the periodogram and its performance may be less affected by the presence of occasional breaks. $^{3}$

\section{An empirical strategy}

As discussed in the Introduction, there are several results in literature about the spurious effects that may arise from the analysis of both stationary data with long memory and non stationary data with structural breaks.

When a series gives the impression of persistence and the estimated value of the long memory parameter $(\hat{d})$ is bigger than zero, there might be the suspicion that this result is due to spurious long memory, provoked by neglected structural breaks.

To overcome this uncertainty it is possible to use an empirical procedure based on the estimation of $d$ after detecting and eliminating the true break points from the series. Indeed this allows (Granger and Hyung, 2004) examining the effects of removing break components on the estimated value of $d$.

More specifically, the following is the simple scheme of the strategy:

- Estimate $d$ in the original series $x_{t}$, obtaining $\hat{d}$

- Estimate the break dates in $x_{t}$ with the BP procedure

- Obtain the break-free series $x_{t}^{\prime}=x_{t}-m_{t}$, where $m_{t}$ is the sample mean of each regime

- Estimate $d$ in $x_{t}^{\prime}$, obtaining $\hat{d}^{\prime}$

If after filtering out the break points, $\hat{d}^{\prime}$ is still approximately close to $\hat{d}$, then long memory should be a true feature of the series and the structural breaks are spurious. If on the contrary, after deleting the break points from the series, $\hat{d}^{\prime}$ tends to zero we can conclude that the long memory is spurious, caused by neglected occasional breaks. ${ }^{4}$

\footnotetext{
${ }^{3}$ Originally, we had considered in our experiment also Smith's estimator but we did not present the results in the tables since its performance seemed to be approximately between the GPH and R-GPH.

${ }^{4}$ For the estimators that possess the asymptotic distribution (e.g. Whitlle and GPH) it is possible to test the null
} 
Because of its empirical nature, this strategy cannot answer the question of whether long memory or structural changes are present for all imaginable frameworks. However it gives helpful indications in some important cases.

\section{Simulation study}

In this Section we describe our twofold Monte Carlo experiment. On the one hand it is devoted to evaluate the performance (in terms of bias and standard errors) of the estimation methods listed in Section 2 in hypothesis of DGP with occasional breaks in mean (in the tables the acronyms rs, av, hi, gph,r-gph, wh stay respectively as R/S, aggregate variance, Higuchi, GPH, R-GPH, Whittle method). On the other hand it aims to show the behaviour of the empirical strategy to distinguish between long memory and occasional-break DGP described in the previous Section.

The functions we use are written in $\mathrm{R}$ language ( $\mathrm{R}$ Development Core Team, 2006) and are available upon request by the authors.

The following are the models we consider in the simulations: ${ }^{5}$

1. DGP1: mean plus noise model, with $p=0.01,0.05,0.1$ and $\sigma_{\eta}^{2}=0.01,0.05,0.1$;

2. DGP2: Markov switching model, with $(p, q)=(0.95,0.95 ; 0.95,0.99 ; 0.99,0.95 ; 0.99,0.99$; $0.999,0.999)$ and $\sigma_{\eta}^{2}=0.1$. In this case the initial state $s_{1}$ is generated by a Bernoulli random variable with $p=0.5$;

3. DGP3: STOPBREAK model, with $\gamma=\left(10^{-5}, 10^{-1}, 1,10,10^{3}\right)$, following Diebold and Inuoe (2001), in order to make comparisons.

4. DGP4: $I(d)$ model, with $d=0.1,0.2,0.3,0.4,0.45$. The error term in normally distributed with zero mean and unit variance.

For each model we consider $\sigma_{\epsilon}^{2}=1$ and $s=1000$ independent realizations. Thus for a given estimation method we obtain $s=1000$ estimated values for $d$. The considered sample sizes are $T=500,1000$ for the first three DGPs and $T=500,1000,2000$ for DGP $4 .{ }^{6}$ All series are generated with 200 additional values to obtain random starting values. The number

hypothesis of true long memory $\left(\hat{d}=\hat{d}^{\prime}\right)$ by extending to this special context the results obtained by Beran and Terrin (1996) and further revised by Horváth and Shao (1999).

${ }^{5}$ We did not consider processes with both long memory and structural breaks since in this case our procedure would clearly detect both phenomena.

${ }^{6}$ For DGP1, DGP2, DGP3 we did not consider $T=2000$ because of the computational burden of the procedure that would have required to much time. 
of frequencies included in the GPH estimator is set $m=\sqrt{T}$ as suggested by Geweke and Porter-Hudack (1983), whereas the number of frequencies triggered out in the R-GPH method is set $l=(m-\tau) / J$ where $\tau=J=4$ as in Lee and Robinson (1996).

In tables 3-6 our results are presented. In each cell of the tables we report in the first row the estimation (standard error in parenthesis) of the long memory parameter $d$ from the original series, whereas in the second row the same estimation from the break-free series.

From the tables we can obtain a conspicuous amount of information regarding the two aims of the simulation study.

Relatively to the first objective, the observation of the first row of the cells in tables 3-5 reveals that long memory is erroneously found whatever estimation method is used. Hence not only the GPH (as already highlighted in the literature) but more in general all the considered estimators are severely upward biased. Whittle and R-GPH estimator exhibit a slightly better performance, in the sense that $\hat{d}$ is often much closer to zero over all the considered DGPs.

Moreover, the perfomance of all methods becomes poorer as the sample size, $T$, and the jump size, $\sigma_{\eta}^{2}$, increase. Indeed, as the series length grows, $T p$ also grows and more breaks are present, that emphasize the non stationary behaviour of the series. Consequently the degree of similarity between occasional-break and long memory processes grows. Similarly, an increase of $\sigma_{\eta}^{2}$ strenghtens the bias of $d$ as the size of the jumps is bigger.

Relatively to the second objective, the comparison between the first and the second row of each cell in tables 3 and 4 reveals a clear change in the estimate before and after removing the break points. The result is particularly interesting when Whittle and R-GPH method are used since in that case the break-free estimation, $\hat{d}^{\prime}$, is approximately around zero. This gives the indication that the long memory found in the original series is in fact spurious and the true DGP should be with occasional breaks.

Some more comments have to be made for table 5 where the variation in the estimation of $d$ after removing the break points is not so evident as in DGP1 and DGP2. In the STOPBREAK process $q_{t}$ is $\frac{\epsilon_{t}}{\gamma+\epsilon_{t}}$ therefore if $\gamma \rightarrow 0, q_{t} \rightarrow 1$ and the process is almost $I(1)$, whereas if $\gamma \rightarrow \infty$, $q_{t} \rightarrow 0$ and the process is $I(0)$. As a consequence, when $\gamma$ does not tend to zero all methods work, since the process is $I(0)$ and it is easier to recognize the non long memory feature. For the smallest values of $\gamma$ the process is so close to $I(1)$ that it is very complicate to identify the DGP. Indeed, in this case it may be more adequate to model the first difference of the series 
rather than looking for structural breaks.

On the other hand, it appears that the empirical procedure is also able to recognize when the DGP is truly long memory. Table 6 shows that the break-free estimation $\left(\hat{d}^{\prime}\right)$ obtained with Whittle and R-GPH methods, is approximately stable around the original estimation $\hat{d}$ and this result improves when $T$ increases.

We observe that there is a slight tendence to underestimate $d$ in the break-free series. This is expected because the implementation of the procedure means subtracting from the series the sample means of each estimated regime and somehow "compressing" the series that looks less persistent. However, when we use Whittle or R-GPH method this is only a negligible effect, whereas for the other estimation methods this phenomenon affects much more the estimates that are often negative. These results are consistent with the findings of Granger and Hyung (2004) about the GPH method.

\section{$6 \quad$ An example on a real time series}

In this Section we illustrate on a real time series how our procedure can be applied in practice. In particular, we apply the empirical strategy to the time series of absolute stock returns of the S\&P 500 from January 2, 1988 to June 15, 2005, with 4292 daily observations. The series of returns is constructed by differencing the log-price index of S\&P 500 .

In several papers this series has been found to possess features typical of long memory data. In particular, Granger and Ding (1995, 1996), Lobato and Savin (1998) suggest a fractionally integrated model. ${ }^{7}$ However, more recently, attempts have been made to explain strong persistence in this series with unaccounted structural breaks (Granger and Hyung, 2004) and the problem is still partially open. On the one hand the only method used to estimate the long memory parameter is the GPH that has been shown to be biased. On the other hand structural change tests are severely biased in presence of long memory. Therefore we take this series as a concrete example of a real problem where our strategy can be used to detect spurious effects.

Figure 1 presents the plot of absolute stock returns as well as the breaks detected by BP procedure. Figure 2 shows the sample autocorrelation function for absolute stock returns that exhibits a slow hyperbolic decay indicating the presence of long memory.

In table 1 are reported the estimates of the long memory parameter in the whole sample

\footnotetext{
${ }^{7}$ The series considered by these authors is in fact the S\&P 500 from January 4, 1928 to October 30, 2002.
} 


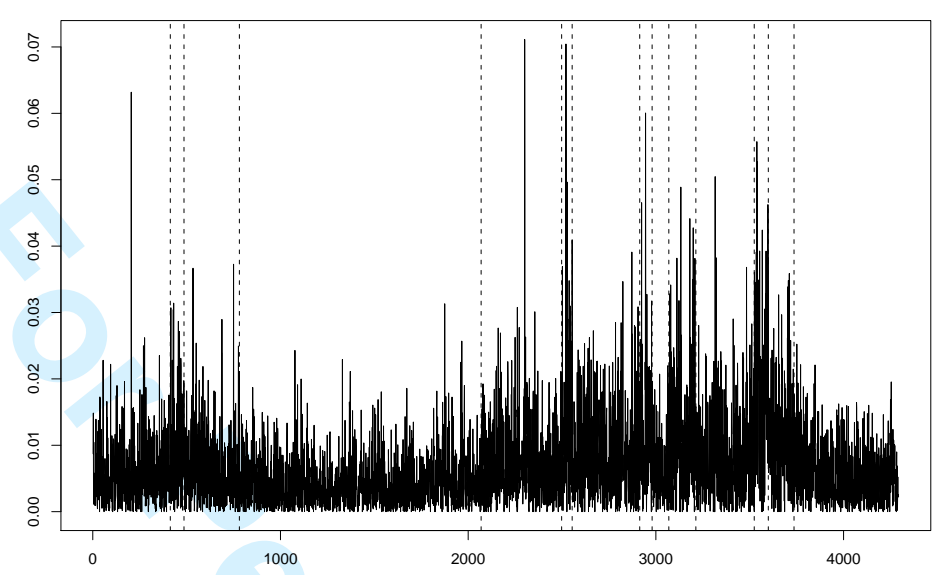

Figure 1: S\&P 500 daily absolute returns and estimated breaks

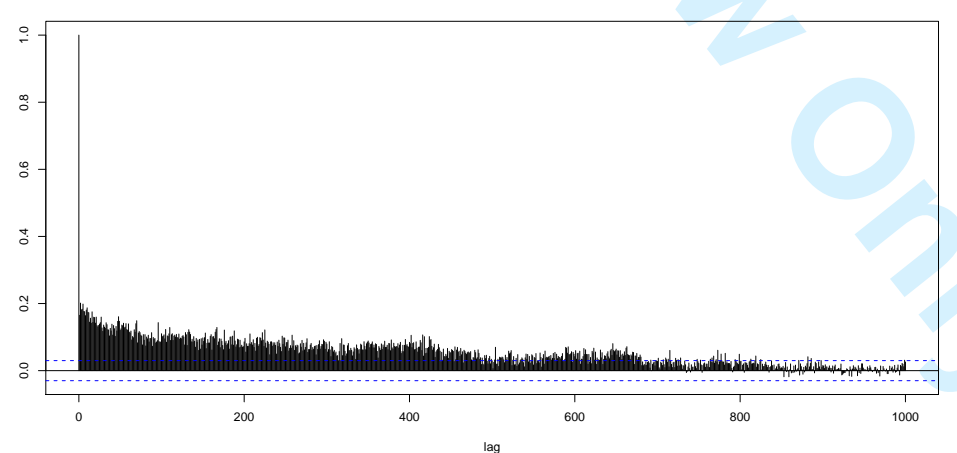

Figure 2: Autocorrelation for S\&P 500 daily absolute returns 
Table 1: Estimation of $d$ in the original and break-free series

\begin{tabular}{ccccccc}
\hline & rs & av & hi & gph & r-gph & wh \\
\hline$\hat{d}$ & 0.815 & 0.397 & 0.506 & 0.534 & 0.437 & 0.160 \\
$\hat{d}^{\prime}$ & 0.014 & -0.107 & -0.131 & -0.161 & 0.050 & 0.015 \\
\hline
\end{tabular}

Table 2: Estimation results for $d$ in the subsamples with Whittle method

\begin{tabular}{cccccccc}
\hline subseries & $\mathbf{1 : 4 1 1}$ & $\mathbf{4 1 2 : 4 8 4}$ & $\mathbf{4 8 5 : 7 7 9}$ & $\mathbf{7 8 0 : 2 0 6 7}$ & $\mathbf{2 0 6 8 : 2 4 9 6}$ & $\mathbf{2 4 9 7 : 2 5 5 2}$ & $\mathbf{2 5 5 3 : 2 9 1 2}$ \\
\hline$\hat{d}$ & 0.000 & 0.000 & 0.000 & 0.048 & 0.079 & 0.044 & 0.000 \\
\hline subseries & $\mathbf{2 9 1 3 : 2 9 7 8}$ & $\mathbf{2 9 7 9 : 3 0 6 7}$ & $\mathbf{3 0 6 8 : 3 2 1 1}$ & $\mathbf{3 2 1 2 : 3 5 2 2}$ & $\mathbf{3 5 2 3 : 3 5 9 7}$ & $\mathbf{3 5 9 8 : 3 7 3 4}$ & $\mathbf{3 7 3 5 : 4 2 9 2}$ \\
\hline$\hat{d}$ & 0.042 & 0.000 & 0.062 & 0.006 & 0.045 & 0.000 & 0.008 \\
\hline
\end{tabular}

before and after the detection of the break points. We can observe that, after removing the break points from the series, the estimated value of the long memory parameter move towards zero whatever estimation method is used. However, as expected from our Monte Carlo study, $\hat{d}^{\prime}$ is very close to zero when computed with Whittle method.

Our conclusion is that the series of $\mathrm{S} \& \mathrm{P} 500$ daily absolute returns is characterized by structural breaks and not by long memory. To give some more evidence on this we estimate (with Whittle method) the long memory parameter in the subseries obtained between breaks and, as expected, they are found to be short memory (table 2).

\section{Conclusions}

In this article we focus on the issue of distinguishing between a time series exhibiting longrange dependence and one with short memory but suffering from occasional structural breaks. Nowadays this is a very interesting topic to which an entire special number of the Journal of Econometrics (vol. 129, issue 1-2, 2005) is devoted.

Firstly, we investigate with an extensive Monte Carlo experiment about the performance of various estimators of the long memory parameter under some occasional-break DGPs. Our findings are that not only the GPH (as well known in literature), but also other estimators of $d$ are severely biased when applied to a series that looks persistent in case of neglected structural 
breaks.

Then, we present an empirical strategy based on the different behaviour of the estimators of the long memory parameter when applied to the original series and its break-free version. From the findings of another simulation study, the procedure, combined to Whittle or R-GPH method, seems in general able to signal the presence of spurious effects. This is a rather crucial point since they are responsible for leading the researcher to erroneously believe that the DGP is long memory when it is in fact with occasional breaks in mean.

It is important to observe that the strategy we describe cannot for all possible circumstances answer the question of whether long memory or structural changes cause the persistent appearance of the series. However it gives indications in various interesting situations.

Many topics are still worthy of investigation, for example the idea of studying models characterized both by long memory and structural breaks. In this direction is the very interesting paper by Gil-Alana (2008) who proposes a simple procedure for determining fractional integration and structural breaks in a unified treatment. With a different approach Ko and Vannucci (2006) describe a wavelet-based bayesian procedure to estimate and locate multiple change points in the long memory parameter of Gaussian autoregressive fractionally integrated moving average models with unknown number of change points.

\section{Acknowledgments}

We thank two anonymous Referees for the insightful comments. 
Table 3: Estimation results for $d$ : DGP1, T=500, 1000

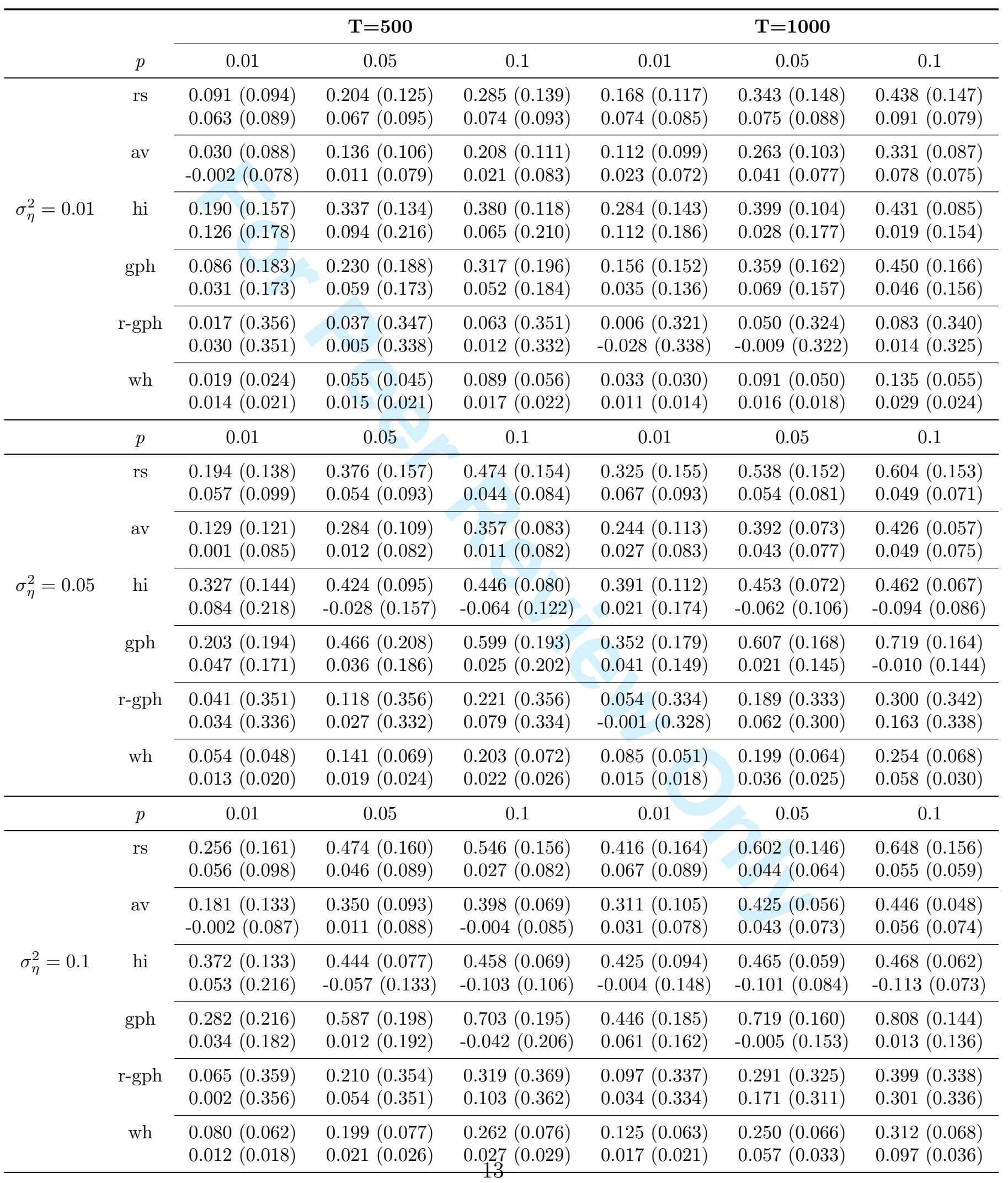


Table 4: Estimation results for $d$ : DGP2, T=500, 1000

\begin{tabular}{|c|c|c|c|c|c|c|}
\hline \multirow[t]{13}{*}{$\mathrm{T}=500$} & $(p, q)$ & $(0.95,0.95)$ & $(0.95,0.99)$ & $(0.99,0.95)$ & $(0.99,0.99)$ & $(0.999,0.999)$ \\
\hline & \multirow[t]{2}{*}{ rs } & $0.679(0.178)$ & $0.558(0.222)$ & $0.694(0.189)$ & $0.651(0.208)$ & $0.439(0.341)$ \\
\hline & & $-0.031(0.068)$ & $0.015(0.084)$ & $-0.064(0.055)$ & $-0.025(0.081)$ & $-0.027(0.079)$ \\
\hline & \multirow[t]{2}{*}{ av } & $0.455(0.046)$ & $0.382(0.119)$ & $0.464(0.040)$ & $0.434(0.087)$ & $0.285(0.235)$ \\
\hline & & $-0.082(0.088)$ & $-0.042(0.084)$ & $-0.121(0.079)$ & $-0.085(0.087)$ & $-0.084(0.091)$ \\
\hline & \multirow[t]{2}{*}{ hi } & $0.474(0.057)$ & $0.463(0.074)$ & $0.477(0.052)$ & $0.468(0.065)$ & $0.328(0.234)$ \\
\hline & & $-0.185(0.090)$ & $-0.084(0.134)$ & $-0.236(0.067)$ & $-0.154(0.121)$ & $-0.132(0.180)$ \\
\hline & \multirow[t]{2}{*}{ gph } & $0.884(0.177)$ & $0.706(0.265)$ & $0.927(0.165)$ & $0.861(0.221)$ & $0.610(0.458)$ \\
\hline & & $-0.238(0.193)$ & $-0.054(0.192)$ & $-0.254(0.165)$ & $-0.166(0.183)$ & $-0.157(0.213)$ \\
\hline & \multirow[t]{2}{*}{ r-gph } & $0.563(0.346)$ & $0.372(0.390)$ & $0.619(0.343)$ & $0.566(0.357)$ & $0.408(0.464)$ \\
\hline & & $0.079(0.332)$ & $0.061(0.359)$ & $0.209(0.345)$ & $0.144(0.370)$ & $0.129(0.381)$ \\
\hline & \multirow[t]{2}{*}{ wh } & $0.436(0.089)$ & $0.282(0.123)$ & $0.499(0.082)$ & $0.419(0.121)$ & $0.308(0.234)$ \\
\hline & & $0.053(0.039)$ & $0.021(0.026)$ & $0.098(0.049)$ & $0.051(0.046)$ & $0.066(0.059)$ \\
\hline \multirow[t]{13}{*}{$\mathrm{T}=1000$} & $(p, q)$ & $(0.95,0.95)$ & $(0.95,0.99)$ & $(0.99,0.95)$ & $(0.99,0.99)$ & $(0.999,0.999)$ \\
\hline & \multirow[t]{2}{*}{ rs } & $0.713(0.180)$ & $0.691(0.176)$ & $0.723(0.207)$ & $0.749(0.186)$ & $0.534(0.341)$ \\
\hline & & $-0.069(0.053)$ & $-0.018(0.076)$ & $-0.091(0.038)$ & $-0.046(0.063)$ & $-0.038(0.076)$ \\
\hline & \multirow[t]{2}{*}{ av } & $0.469(0.035)$ & $0.447(0.060)$ & $0.474(0.034)$ & $0.467(0.040)$ & $0.342(0.212)$ \\
\hline & & $-0.139(0.072)$ & $-0.060(0.079)$ & $-0.177(0.058)$ & $-0.117(0.074)$ & $-0.120(0.089)$ \\
\hline & \multirow[t]{2}{*}{ hi } & $0.475(0.054)$ & $0.473(0.059)$ & $0.479(0.052)$ & $0.480(0.046)$ & $0.371(0.204)$ \\
\hline & & $-0.246(0.064)$ & $-0.142(0.098)$ & $-0.281(0.049)$ & $-0.200(0.089)$ & $-0.156(0.167)$ \\
\hline & \multirow[t]{2}{*}{ gph } & $0.942(0.128)$ & $0.838(0.177)$ & $0.963(0.133)$ & $0.942(0.150)$ & $0.705(0.421)$ \\
\hline & & $-0.124(0.134)$ & $-0.070(0.154)$ & $-0.139(0.143)$ & $-0.110(0.154)$ & $-0.092(0.155)$ \\
\hline & \multirow[t]{2}{*}{ r-gph } & $0.611(0.305)$ & $0.464(0.353)$ & $0.654(0.302)$ & $0.615(0.324)$ & $0.474(0.406)$ \\
\hline & & $0.421(0.339)$ & $0.171(0.375)$ & $0.485(0.354)$ & $0.421(0.395)$ & $0.413(0.398)$ \\
\hline & \multirow[t]{2}{*}{ wh } & $0.472(0.075)$ & $0.348(0.095)$ & $0.532(0.071)$ & $0.469(0.086)$ & $0.367(0.219)$ \\
\hline & & $0.099(0.041)$ & $0.031(0.028)$ & $0.164(0.037)$ & $0.102(0.046)$ & $0.099(0.073)$ \\
\hline
\end{tabular}


Table 5: Estimation results for $d$ : DGP3, $\mathrm{T}=500,1000$

\begin{tabular}{|c|c|c|c|c|c|c|}
\hline \multirow{13}{*}{$\mathrm{T}=500$} & $\gamma$ & $10^{-5}$ & $10^{-1}$ & 1 & 10 & $10^{3}$ \\
\hline & \multirow[t]{2}{*}{ rs } & $0.719(0.308)$ & $0.731(0.330)$ & $0.714(0.285)$ & $0.709(0.191)$ & $0.044(0.085)$ \\
\hline & & $-0.093(0.043)$ & $-0.092(0.041)$ & $-0.089(0.046)$ & $-0.059(0.060)$ & $0.037(0.086)$ \\
\hline & \multirow[t]{2}{*}{ av } & $0.476(0.030)$ & $0.478(0.033)$ & $0.475(0.033)$ & $0.463(0.041)$ & $-0.012(0.075)$ \\
\hline & & $-0.204(0.058)$ & $-0.207(0.058)$ & $-0.192(0.060)$ & $-0.116(0.084)$ & $-0.018(0.074)$ \\
\hline & \multirow[t]{2}{*}{ hi } & $0.476(0.051)$ & $0.479(0.049)$ & $0.479(0.047)$ & $0.475(0.052)$ & $0.076(0.132)$ \\
\hline & & $-0.279(0.042)$ & $-0.282(0.042)$ & $-0.278(0.042)$ & $-0.230(0.074)$ & $0.069(0.131)$ \\
\hline & \multirow[t]{2}{*}{ gph } & $0.989(0.162)$ & $0.994(0.164)$ & $0.997(0.157)$ & $0.924(0.154)$ & $0.011(0.172)$ \\
\hline & & $-0.615(0.155)$ & $-0.609(0.155)$ & $-0.508(0.143)$ & $-0.231(0.172)$ & $0.017(0.162)$ \\
\hline & \multirow{2}{*}{ r-gph } & $0.747(0.328)$ & $0.734(0.318)$ & $0.749(0.319)$ & $0.624(0.327)$ & $-0.005(0.344)$ \\
\hline & & $-0.213(0.374)$ & $-0.247(0.362)$ & $-0.066(0.363)$ & $0.267(0.368)$ & $-0.017(0.353)$ \\
\hline & \multirow[t]{2}{*}{ wh } & $0.982(0.023)$ & $0.958(0.033)$ & $0.825(0.059)$ & $0.485(0.082)$ & $0.012(0.018)$ \\
\hline & & $0.695(0.051)$ & $0.657(0.051)$ & $0.472(0.056)$ & $0.080(0.046)$ & $0.009(0.017)$ \\
\hline \multirow[t]{13}{*}{$T=1000$} & $\gamma$ & $10^{-5}$ & $10^{-1}$ & 1 & 10 & $10^{3}$ \\
\hline & \multirow[t]{2}{*}{ rs } & $0.729(0.348)$ & $0.707(0.339)$ & $0.731(0.302)$ & $0.728(0.199)$ & $0.061(0.084)$ \\
\hline & & $-0.057(0.046)$ & $-0.055(0.048)$ & $-0.010(0.045)$ & $0.128(0.060)$ & $0.044(0.081)$ \\
\hline & \multirow[t]{2}{*}{ av } & $0.478(0.029)$ & $0.478(0.029)$ & $0.478(0.046)$ & $0.473(0.031)$ & $0.011(0.073)$ \\
\hline & & $-0.163(0.049)$ & $-0.163(0.053)$ & $-0.104(0.061)$ & $0.111(0.069)$ & $-0.001(0.068)$ \\
\hline & \multirow[t]{2}{*}{ hi } & $0.477(0.052)$ & $0.476(0.052)$ & $0.479(0.046)$ & $0.477(0.054)$ & $0.147(0.144)$ \\
\hline & & $-0.271(0.036)$ & $-0.267(0.039)$ & $-0.229(0.046)$ & $-0.087(0.068)$ & $0.137(0.155)$ \\
\hline & \multirow[t]{2}{*}{ gph } & $0.997(0.125)$ & $1.001(0.128)$ & $1.001(0.127)$ & $0.962(0.125)$ & $0.045(0.142)$ \\
\hline & & $-0.590(0.111)$ & $-0.597(0.118)$ & $-0.591(0.122)$ & $-0.279(0.133)$ & $0.023(0.138)$ \\
\hline & \multirow{2}{*}{ r-gph } & $0.735(0.303)$ & $0.720(0.293)$ & $0.723(0.296)$ & $0.661(0.293)$ & $0.0134(0.330)$ \\
\hline & & $-0.188(0.349)$ & $-0.213(0.354)$ & $-0.177(0.356)$ & $0.354(0.316)$ & $-0.008(0.321)$ \\
\hline & \multirow[t]{2}{*}{ wh } & $0.987(0.015)$ & $0.965(0.025)$ & $0.834(0.049)$ & $0.514(0.069)$ & $0.010(0.015)$ \\
\hline & & $0.825(0.033)$ & $0.793(0.033)$ & $0.656(0.036)$ & $0.324(0.045)$ & $0.009(0.014)$ \\
\hline
\end{tabular}


Table 6: Estimation results for $d$ : DGP $\operatorname{ARFIMA(0,d,0),~T=500,~1000,~} 2000$

\begin{tabular}{|c|c|c|c|c|c|c|}
\hline \multirow[t]{13}{*}{$\mathrm{T}=500$} & $d$ & 0.1 & 0.2 & 0.3 & 0.4 & 0.45 \\
\hline & rs & $0.117(0.089)$ & $0.201(0.103)$ & $0.272(0.111)$ & $0.360(0.120)$ & $0.393(0.129)$ \\
\hline & & $0.075(0.087)$ & $0.048(0.077)$ & $0.000(0.069)$ & $-0.033(0.056)$ & $-0.041(0.054)$ \\
\hline & av & $0.068(0.074)$ & $0.153(0.075)$ & $0.216(0.074)$ & $0.289(0.073)$ & $0.317(0.069)$ \\
\hline & & $0.024(0.076)$ & $0.011(0.078)$ & $-0.037(0.073)$ & $-0.080(0.069)$ & $-0.095(0.074)$ \\
\hline & hi & $0.074(0.107)$ & $0.184(0.109)$ & $0.262(0.117)$ & $0.369(0.104)$ & $0.417(0.098)$ \\
\hline & & $0.017(0.122)$ & $-0.029(0.147)$ & $-0.138(0.089)$ & $-0.194(0.072)$ & $-0.208(0.071)$ \\
\hline & gph & $0.117(0.162)$ & $0.197(0.167)$ & $0.315(0.175)$ & $0.408(0.178)$ & $0.481(0.166)$ \\
\hline & & $0.095(0.165)$ & $0.101(0.172)$ & $0.080(0.183)$ & $-0.014(0.178)$ & $-0.085(0.183)$ \\
\hline & r-gph & $0.082(0.350)$ & $0.165(0.335)$ & $0.241(0.371)$ & $0.284(0.369)$ & $0.371(0.340)$ \\
\hline & & $0.079(0.347)$ & $0.162(0.336)$ & $0.217(0.364)$ & $0.244(0.372)$ & $0.311(0.358)$ \\
\hline & wh & $0.093(0.036)$ & $0.194(0.033)$ & $0.291(0.036)$ & $0.396(0.039)$ & $0.446(0.036)$ \\
\hline & & $0.079(0.037)$ & $0.148(0.041)$ & $0.215(0.044)$ & $0.291(0.046)$ & $0.332(0.047)$ \\
\hline \multirow[t]{13}{*}{$T=1000$} & $d$ & 0.1 & 0.2 & 0.3 & 0.4 & 0.45 \\
\hline & $\mathrm{rs}$ & $0.101(0.089)$ & $0.199(0.099)$ & $0.286(0.109)$ & $0.354(0.118)$ & $0.399(0.114)$ \\
\hline & & $0.062(0.077)$ & $0.021(0.067)$ & $-0.017(0.054)$ & $-0.034(0.048)$ & $-0.032(0.042)$ \\
\hline & av & $0.063(0.067)$ & $0.155(0.071)$ & $0.235(0.071)$ & $0.292(0.068)$ & $0.325(0.062)$ \\
\hline & & $0.027(0.067)$ & $-0.007(0.066)$ & $-0.056(0.067)$ & $-0.089(0.061)$ & $-0.099(0.060)$ \\
\hline & hi & $0.080(0.111)$ & $0.181(0.110)$ & $0.275(0.106)$ & $0.359(0.109)$ & $0.401(0.105)$ \\
\hline & & $0.024(0.118)$ & $-0.085(0.122)$ & $-0.169(0.079)$ & $-0.216(0.062)$ & $-0.220(0.060)$ \\
\hline & gph & $0.106(0.139)$ & $0.201(0.124)$ & $0.306(0.134)$ & $0.404(0.132)$ & $0.458(0.138)$ \\
\hline & & $0.088(0.139)$ & $0.096(0.141)$ & $0.059(0.153)$ & $-0.010(0.153)$ & $-0.097(0.153)$ \\
\hline & r-gph & $0.089(0.354)$ & $0.121(0.330)$ & $0.198(0.350)$ & $0.288(0.326)$ & $0.316(0.352)$ \\
\hline & & $0.087(0.355)$ & $0.108(0.334)$ & $0.186(0.347)$ & $0.243(0.318)$ & $0.277(0.340)$ \\
\hline & wh & $0.094(0.025)$ & $0.196(0.025)$ & $0.296(0.027)$ & $0.399(0.027)$ & $0.449(0.026)$ \\
\hline & & $0.087(0.024)$ & $0.165(0.027)$ & $0.242(0.031)$ & $0.333(0.032)$ & $0.379(0.032)$ \\
\hline \multirow[t]{13}{*}{$\mathrm{T}=\mathbf{2 0 0 0}$} & $d$ & 0.1 & 0.2 & 0.3 & 0.4 & 0.45 \\
\hline & $\mathrm{rs}$ & $0.110(0.084)$ & $0.196(0.092)$ & $0.279(0.096)$ & $0.369(0.110)$ & $0.407(0.117)$ \\
\hline & & $0.069(0.072)$ & $0.052(0.066)$ & $0.058(0.060)$ & $0.091(0.065)$ & $0.112(0.062)$ \\
\hline & av & $0.074(0.067)$ & $0.159(0.068)$ & $0.232(0.065)$ & $0.304(0.065)$ & $0.333(0.059)$ \\
\hline & & $0.041(0.065)$ & $0.044(0.065)$ & $0.054(0.067)$ & $0.095(0.072)$ & $0.120(0.069)$ \\
\hline & hi & $0.083(0.104)$ & $0.182(0.109)$ & $0.273(0.106)$ & $0.361(0.105)$ & $0.414(0.092)$ \\
\hline & & $0.028(0.112)$ & $-0.046(0.115)$ & $-0.095(0.076)$ & $-0.093(0.069)$ & $-0.085(0.071)$ \\
\hline & gph & $0.110(0.113)$ & $0.198(0.108)$ & $0.303(0.117)$ & $0.396(0.120)$ & $0.479(0.112)$ \\
\hline & & $0.093(0.111)$ & $0.117(0.112)$ & $0.099(0.121)$ & $0.063(0.126)$ & $0.018(0.122)$ \\
\hline & r-gph & $0.088(0.355)$ & $0.144(0.348)$ & $0.218(0.365)$ & $0.263(0.331)$ & $0.332(0.341)$ \\
\hline & & $0.086(0.355)$ & $0.140(0.347)$ & $0.206(0.364)$ & $0.240(0.337)$ & $0.300(0.358)$ \\
\hline & wh & $0.097(0.018)$ & $0.198(0.017)$ & $0.298(0.018)$ & $0.399(0.018)$ & $0.450(0.017)$ \\
\hline & & $0.093(0.018)$ & $0.185(0.018)$ & $0.280(0.019)$ & $0.378(0.019)$ & $0.428(0.019)$ \\
\hline
\end{tabular}




\section{References}

Bai, J. and Perron, P., 1998, Estimating and testing linear models with multiple structural changes. Econometrica, 66, 47-78.

Bai, J. and Perron, P., 2003, Computation and analysis of multiple structural change models. Journal of Applied Econometrics, 18, 1-22.

Beran, J. and Terrin, N., 1996, Testing for a change of the long-memory parameter. Biometrika, 83, 627-638.

Berkes, I., Horváth, L., Kokosvka, P. and Shao, Q.M., 2006, On discriminating between longrange dependence and changes in mean. The Annals of Statistics, 34, 1140-1165.

Beran, J., 1994, Statistics for Long-Memory Processes. Chapman and Hall, London.

Chen, C. and Tiao, G.C., 1990, Random level-shift time series models, ARIMA approximations, and level-shift detection. Journal of Business and Economics Statistics, 8, 83-97.

Craigmile, P.F., Guttorp, P. and Percival, D.B., 2005 Wavelet-based parameter estimation for polynomial contaminated fractionally differenced processes. IEEE Transactions on signal processing, 53, 3151-3161.

Diebold, F.X. and Inoue, A., 2001, Long memory and regime switching. Journal of Econometrics, 105, 131-159.

Dolado, J.J, Gonzalo, J. and Mayoral, L., 2004, A Simple Test of Long-Memory vs. Structural Breaks in the Time Domain: What is What? Universidad Carlos III de Madrid (mimeo).

Engle, R.F. and Smith, A.D., 1999, Stochastic permanent breaks. The Review of Economics and Statistics, 81, 553-574.

Fox, R. and Taqqu, M.S., 1986, Large-sample properties of parameter estimates for strongly dependent stationary Gaussian time series. The Annals of Statistics, 14, 517-532.

Gabriel, V.J. and Martins, L.F., 2004, On the forecasting ability of ARFIMA models when infrequent breaks occur. Econometrics Journal, 7, 455-475.

Geweke, J. and Porter-Hudack S., 1983, The Estimation and Application of Long-Memory Time Series Models. J.T.S.A., 4, (1983) 221-237.

Gil-Alana, L.A., 2004, Structural change and the order of integration in univariate time series. Computational Economics, 23, 239-254. 
Gil-Alana, L.A., 2008, Fractional integration and structural breaks at unknown periods of time. Journal of time series analysis, 29, 163-185.

Giraitis, L., Kokoszka, P.S. and Leipus R., 2001, Testing for long memory in the presence of a general trend. Journal of Applied probability, 38, 1033-1054.

Granger, C.W.J. and Ding, Z., 1995, Some properties of absolute returns. An alternative measure of risk. Annales d'Economie et de Statistique, 40, 67-91.

Granger, C.W.J. and Ding, Z., 1996, Varieties of long memory models. Journal of Econometrics, $\mathbf{7 3}, 61-78$.

Granger, C.W.J. and Joyeux, R., 1980, An introduction to long-range time series models and fractional differencing. Journal of Time Series Analysis, 1, 15-30.

Granger, C.W.J. and Hyung, N., 2004, Occasional structural breaks and long memory with an application to the S\&P500 absolute stock returns. Journal of Empirical Finance, 11, $399-421$.

Hamilton, J.D., 1989, A new approach to the economic analysis of nonstationary time series and the business cycle. Econometrica, 57, 357-384.

Higuchi, T., 1988, Approach to an irregular time series on the basis of the fractal theory. Physica D, 31, 277-283.

Horváth, L. and Shao Q-M., 1999, Limit theorems for quadratic forms with applications to Whittle's estimate. The Annals of Applied Probability, 9, 146-187.

Hosking, J.R.M., 1981, Fractional differencing. Biometrika, 68, 165-176.

Hsu, C., 2005, Long memory and structural changes: an empirical examination on inflation rates. Economics Letters, 88, 289-294.

Hurst, H.E., 1951, Long-term storage capacity of reservoirs. Transactions of the American Society of Civil Engineers, 116, 770-799.

Jensen, M., 1999, Using wavelets to obtain a consistent ordinary least square estimator of the long-memory parameter. Journal of Forecasting, 18, 17-32.

Ko, K. and Vannucci, M., 2006, Bayesian wavelet-based methods for the detection of multiple changes of the long memory parameter. IEEE Transactions on Signal Processing, 54, 4461-4470 
Kuan, C.M. and Hsu, C.C., 1998, Change point estimation of fractionally Integrated Processes. Journal of Time Series Analysis, 19, 693-708.

Kunsch, H., 1987, Statistical aspects of self-similar processes. In Proceedings of the First World Congress of the Bernoulli Society (Yu. Prokhorov and V. V. Sazanov, eds.) 1 67-74. VNU Science Press, Utrecht.

Lee, D.K.C. and Robinson, P.M., 1996, Semiparametric Exploration of Long Memory in Stock Prices. Journal of Statistical Planning and Inference, 50, 155-174.

Lobato, I.N. and Robinson, P.M., 1998, A non parametric test for I(0). Review of Economic Studies, 65, 475-495.

Lobato, I.N. and Savin, N.E., 1998, Real and spurious long-memory properties of stock-market data. Journal of Business and Economic Statistics, 16, 261-268.

Mandelbrot, B.B., 1972, Statistical Methodology for nonperiodic cycles: from the Covariance to R/S Analysis. Ann. Economic. Soc. Measurement, 1, (1972) 259-290.

Mandelbrot, B.B., 1975, Limit theorems of the self-normalized range for weakly and strongly dependent processes. Z. Wahr. verw. Geb., 31, (1975) 271-285.

Mandelbrot, B.B. and Taqqu, M.S., 1979, Robust analysis of long-run serial correlation. Proceedings of the 42nd Session of the International Statistical Institute, Manila, 1979. Bulletin of the International Statistical Institute, Vol. 48, Book 2, 69-104.

Morana, C. and Beltratti, A., 2004, Structural change and long-range dependence in volatility of exchange rates: either, neither or both? Journal of Empirical Finance, 11, 629-658.

Ohanissian, A., Russell, J.R. and Tsay, R.S., 2004, True or spurious long memory? A new test. University of Chicago (mimeo).

Palma, W., 2007, Long-Memory Time series: Theory and methods Wiley

Percival, D.B., Walden A.T., 2007, Wavelets Methods for Time Series Analysis Cambridge University Press

Perron, P. and Qu, Z., 2006, An analytical evaluation of the log-periodogram estimate in the presence of level shifts. and its implications for stock returns volatility. mimeo.

R Development Core Team, 2006, R: A Language and Environment for Statistical Computing. R Foundation for Statistical Computing,Vienna, Austria. url = http://www.R-project.org 
Robinson P. M., 1995a, Log-periodogram regression of time series with long range dependence. The Annals of Statistics, 23, 1048-1072.

Robinson, P. M., 1995b, Gaussian semiparametric estimation of long range dependence. Annals of Statistics,23, 1630-1661.

Smith A., 2005, Level Shifts and the Illusion of Long Memory in Economic Time Series. Journal of Business and Economic Statistics, 23, 321-335.

Taqqu M., Teverovsky V. and Willinger W., 1995, Estimators for long-range dependance: an empirical study. Fractals, 3, 785-798.

Teyssiere, G. and Abry P., 2006 Wavelet analysis of nonlinear long-range dependent processes. Applications to financial time series. in Long memory in economics, ed. Teyssiere, G. and Kirman A.P., Springer, 173-238.

Wang, Y., 1999, Change-points via wavelets for indirect data. Statistica Sinica, 9, 103-117. 


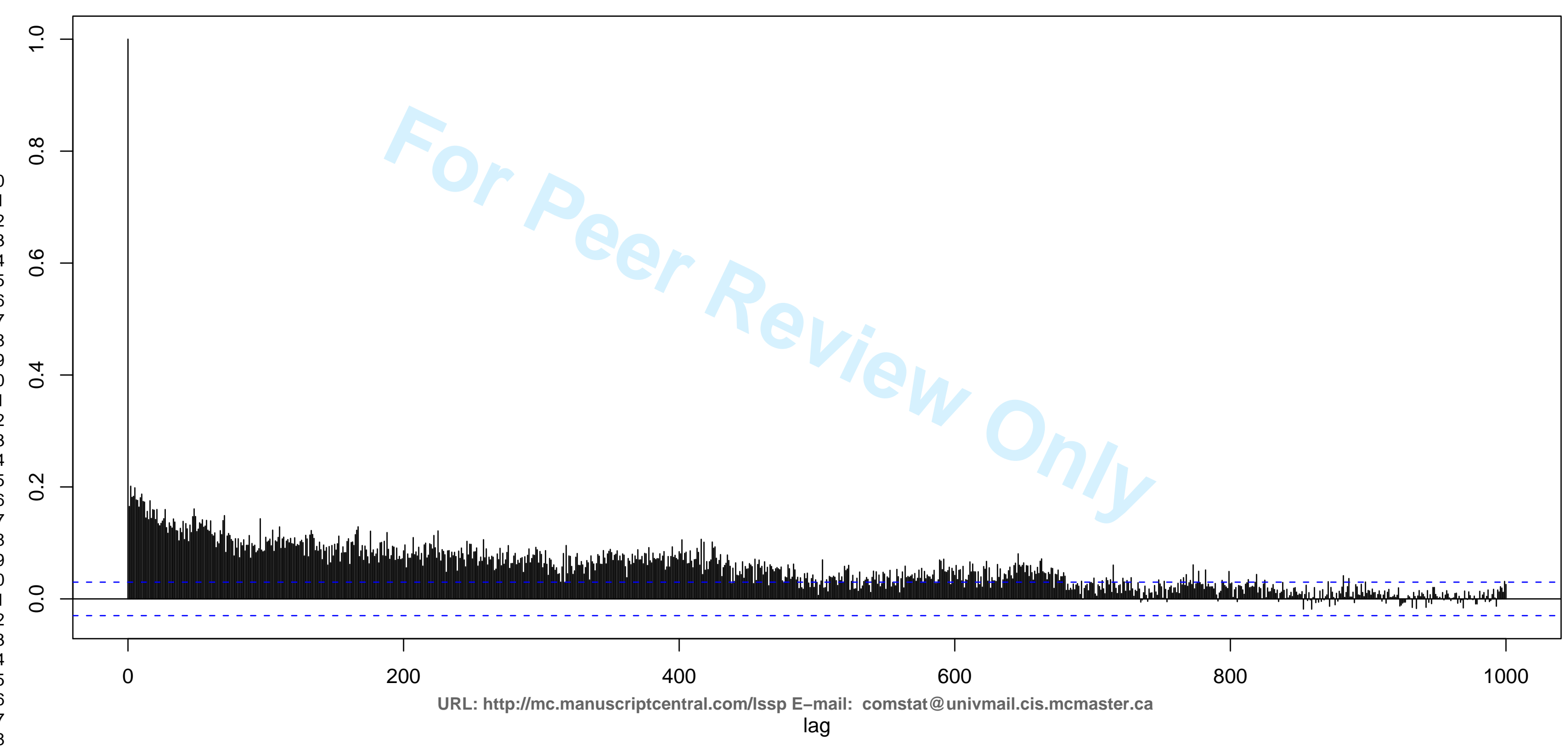




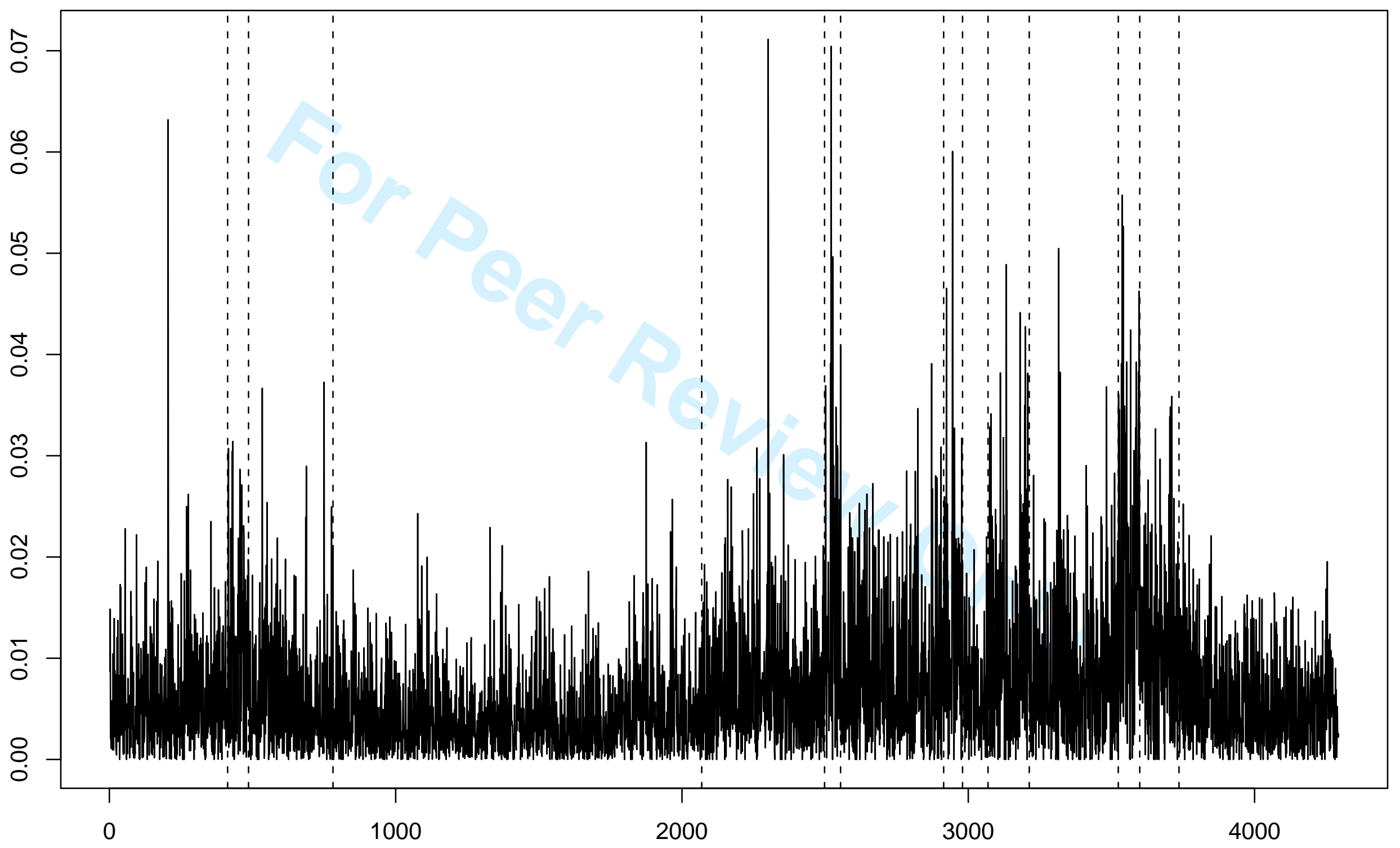

URL: http://mc.manuscriptcentral.com/Issp E-mail: comstat@univmail.cis.mcmaster.ca 